\title{
Incremental Value of SPECT/CT in the Detection of Femoral Head Viability in Slipped Capital Femoral Epiphysis
}

\author{
Andreia DosSantos ${ }^{1}$, Noel Macalintal ${ }^{1}$, Yassine Bouchareb ${ }^{1,2}$, Emily Joel ${ }^{1}$, Hikmat Jan ${ }^{1}$, and Athar Haroon ${ }^{1}$ \\ ${ }^{1}$ Barts Health NHS Trust, Nuclear Medicine Department, London, United Kingdom; and ${ }^{2}$ Queen Mary University, London, United Kingdom
}

In this article, we discuss the role of $99 \mathrm{~m}$ Tc-methylene diphosphonate SPECT/CT in 2 cases of slipped capital femoral epiphysis. We describe the incremental value of SPECT/CT in determining the viability of the femoral head and the implications in management of patients with slipped epiphysis.

Key Words: SPECT/CT; viable; SUFE; SCFE; avascular necrosis

J Nucl Med Technol 2018; 46:153-154

DOI: 10.2967/jnmt.117.202283

\section{A \\ vascular necrosis in the region of femoral epiphysis is a disease in which there is ischemic death of the cellular ele- ments of bone and marrow. The predisposing causes include dislocation of the hip, femoral neck fracture, corticosteroid use, alcoholism, collagen vascular disease, hemoglobinopathies, Gaucher disease, Caisson disease, and some skeletal dysplasias. The purpose of the current teaching case study is to evaluate the role of SPECT/CT imaging in detecting viability of the femoral head after surgery for slipped capital femoral epiphysis.}

\section{CASE REPORTS}

\section{Case 1}

A 14-y-old boy presented with left hip pain. Radiographs showed a slip of the left capital femoral epiphysis, which was further confirmed on MRI (Fig. 1A). The patient underwent bilateral surgical fixation (pinning) of the femoral heads. Postoperatively, a ${ }^{99 m} \mathrm{Tc}-m e t h y l e n e ~ d i p h o s p h-$ onate bone scan (550 MBq, intravenously) and SPECT/CT showed tracer uptake bilaterally at the femoral heads (Fig. 1B). There was satisfactory alignment of the epiphysis and screws. Blood-pool and osteoblastic activity on the affected side was suggestive of a perfused and viable femoral head after surgery. A follow-up radiograph obtained $2 \mathrm{y}$ after the operation showed a smooth outline of the femoral head and satisfactory alignment of the epiphysis bilaterally (Fig. 1C).

\footnotetext{
Received Sep. 15, 2017; revision accepted Dec. 11, 2017.

For correspondence or reprints contact: Athar Haroon, MBBS, FRCR, Consultant Radionuclide Radiologist, Department of Nuclear Medicine, St. Bartholomew's Hospital, West Smithfield EC1A 7BE London, U.K.

E-mail: atharharoon@yahoo.com

Published online Feb. 2, 2018

COPYRIGHT (C) 2018 by the Society of Nuclear Medicine and Molecular Imaging
}

\section{Case 2}

An 8-y-old girl presented with a history of left hip pain. Radiographs showed a moderate posteromedial slip of the left capital femoral epiphysis. MRI of the hips, in addition to showing the slip, demonstrated associated inflammatory changes, with a small effusion at the left hip joint and marrow edema at the physeal surface of the left capital femoral epiphysis (Fig. 2A). The patient underwent screw fixation of the hips. Postoperatively, early blood-pool static images after intravenous injection of $525 \mathrm{MBq}$ of ${ }^{99 \mathrm{~m}} \mathrm{Tc}$-methylene diphosphonate showed absence of tracer in the region of the left femoral head. Three hours after injection, static images showed photopenia in the left femoral head. Three-dimensional SPECT/CT images confirmed absence of uptake in the left capital femoral epiphysis (Fig. 2B). Radiography 2 y after screw fixation showed avascular necrosis of the left femoral head with irregularity, flattening, and sclerosis of the femoral head (Fig. 2C).

\section{DISCUSSION}

Slipped capital femoral epiphysis is defined as a posterior and inferior slippage of the proximal femoral epiphysis (1).

We have found SPECT/CT helpful in determining whether the femoral head is viable in young patients, and this article presents two such cases. There are many advantages to SPECT/CT in this clinical situation. MRI is the imaging investigation of choice in young patients but is contraindicated when metal internal fixation devices and screws are present. SPECT/CT is a valid alternative that can reveal the photopenic femoral head while overcoming the planar imaging artifacts caused by superimposition of overlying structures. SPECT/CT helps by providing an anatomic correlation and localization for a functional abnormality seen on a bone scan. In a study by Krishan et al. (2), diagnostic accuracy was $67 \%$ for bone scans, $78 \%$ for SPECT, and $95 \%$ for SPECT/CT. The area under the receiver-operating-characteristic curve was 0.919 for SPECT/ CT, 0.76 for SPECT, and 0.56 for planar imaging. The CT component of the SPECT/CT study improves the detection of subtle collapse, which can easily be missed on planar images. Alternative diagnoses for the hip pain can also be shown, such as impingement and nail protrusion postoperatively.

Gelfand et al. (3) have described 3 patterns of bone scan uptake related to slipped capital femoral epiphysis: increased uptake in the physis and adjacent proximal metaphysis, decreased uptake in the femoral head, and a postsurgical change in the scintigraphic appearance after internal fixation. The activity 

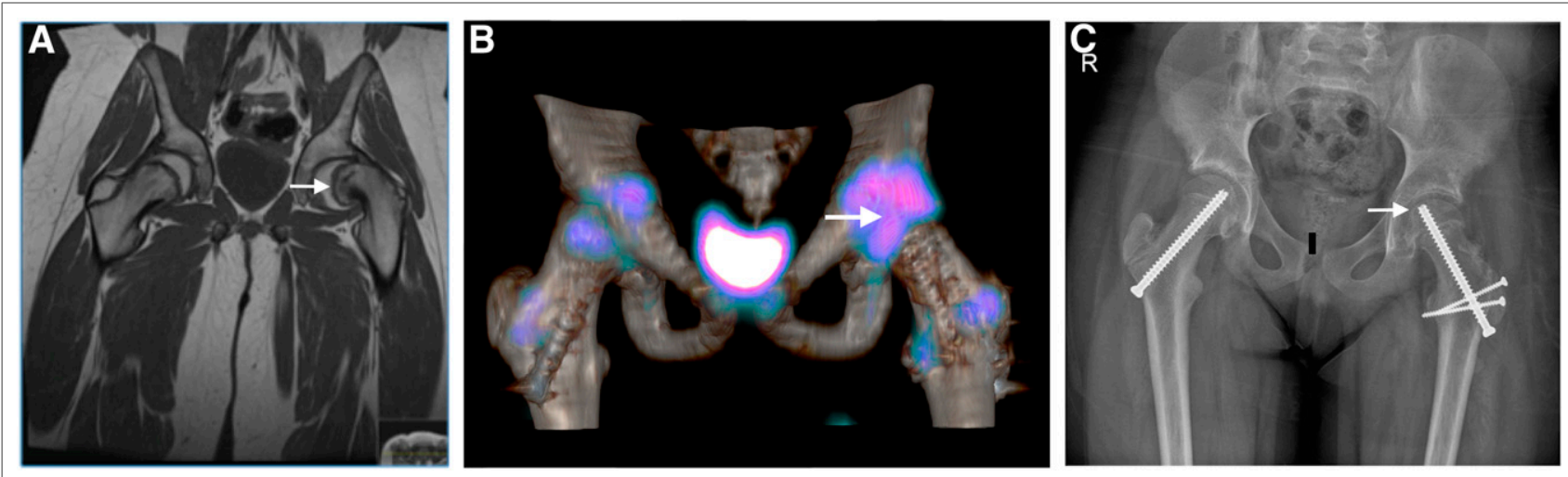

FIGURE 1. (A) Coronal T1-weighted MR image showing left slipped capital femoral epiphysis (arrow). (B) Axial fused 3-dimensional SPECT/CT image showing uptake in femoral head (arrow), with satisfactory appearance of articular surface. (C) Radiograph obtained at $2-y$ follow-up showing smooth outline of left femoral head (arrow).
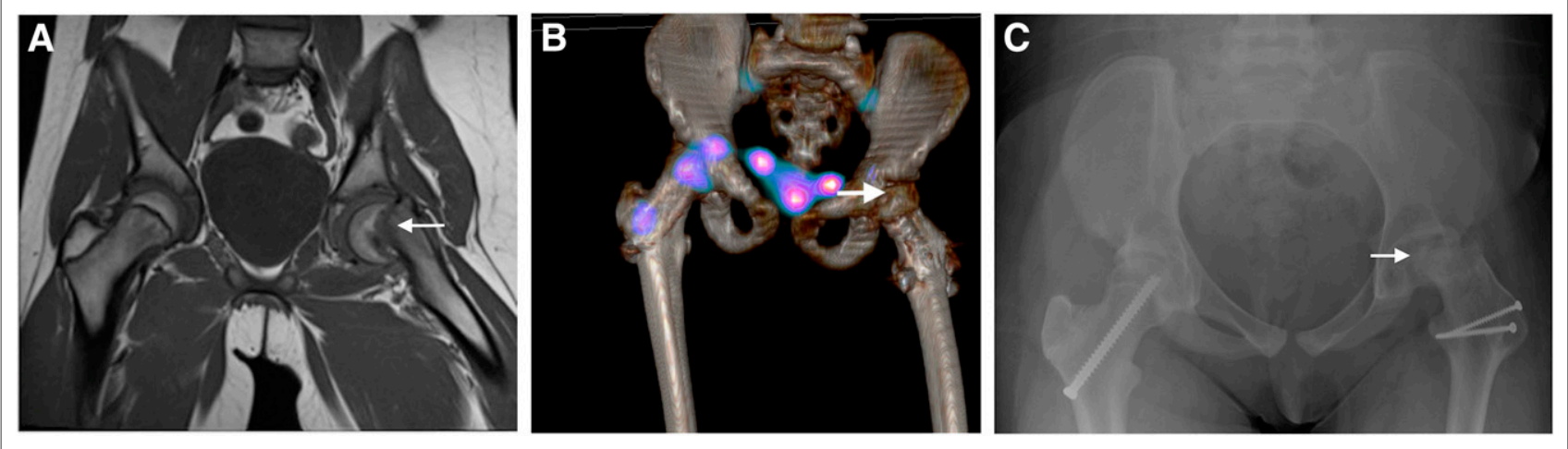

FIGURE 2. (A) Coronal T1-weighted MR image showing left slipped capital epiphysis (arrow). (B) Postoperative axial fused 3-dimensional SPECT/CT image showing absence of uptake in left femoral head (arrow), in keeping with nonviable region. (C) Radiograph obtained at 2-y follow-up showing collapse of left femoral head (arrow) at same site where 3-dimensional SPECT/CT showed nonviable femoral head.

may approach the level of radiopharmaceutical uptake found in the adjacent femoral neck and head.

Loder et al. (4) introduced the concept of instability and recognized 2 types of slips that have clinical, radiologic, and functional significance. In a series of 55 patients with slipped capital femoral epiphysis, Loder et al. showed that avascular necrosis developed in $47 \%$ of the unstable slips but in none of the stable slips.

SPECT/CT helps achieve the main treatment goals for slipped capital femoral epiphysis (1): preventing further epiphyseal displacement until physeal closure, avoiding complications such as avascular necrosis and chondrolysis, and maintaining adequate hip function.

Complications of slipped capital femoral epiphysis include chondrolysis, avascular necrosis, premature osteoarthritis, slip progression despite surgery, loosening, infection, fracture, migration of metal implants, femoral head rotation, subtrochanteric fracture, and joint infection (5).

\section{CONCLUSION}

Bone scintigraphy and SPECT/CT are valuable tools to detect viability of the femoral head after surgery. Patients with reduced viability are offered calcium supplements (bisphosphonates) to enhance bone healing. Bone scans coupled with SPECT/CT facilitates triage of patients who will benefit from medical treatment after surgery for slipped capital femoral epiphysis.

\section{DISCLOSURE}

No potential conflict of interest relevant to this article was reported.

\section{REFERENCES}

1. Mulgrew E, Wells-Cole S, Ali F, Joshy S, Siddique I, Zenios M. Single screw fixation in stable and unstable slipped upper femoral epiphysis. J Pediatr Orthop B. 2011;20:147-151.

2. Agarwal KK, Mukherjee A, Sharma P, Bal C, Kumar R. Incremental value of ${ }^{99 \mathrm{~m} T c-M D P}$ hybrid SPECT/CT over planar scintigraphy and SPECT in avascular necrosis of the femoral head. Nucl Med Commun. 2015;36:1055-1062.

3. Gelfand MJ, Strife JL, Graham EJ, Crawford AH. Bone scintigraphy in slipped capital femoral epiphysis. Clin Nucl Med. 1983;8:613-615.

4. Loder RT, Richards BS, Shapiro PS, Reznick LR, Aronson DD. Acute slipped capital femoral epiphysis: the importance of physeal stability. J Bone Joint Surg Am. 1993;75:1134-1140.

5. Tins B, Cassar-Pullicino V, McCall I. Slipped upper femoral epiphysis: imaging of complications after treatment. Clin Radiol. 2008;63:27-40. 